\title{
Eigenmode Frequencies Calculations in the Charge-Density-Wave States of $2 \mathrm{H}-\mathrm{TaSe}_{2}$.
}

\author{
A. Ribeiro Filho, E. M. do Nascimento and \\ D. S. de Vasconcelos \\ Instituto de Física, Universidade Federal da Bahia \\ Campus de Ondina, 40.210-340, Salvador, Ba, Brazil
}

Received 8 June, 1998. Revised version received on 17 August, 1998

We have performed some calculations involving two eigenmode frequencies of $2 \mathrm{H}-\mathrm{TaSe}_{2}$. We have obtained a good comparison with some well established Raman scattering data for the commensurate and incommensurate charge-density-wave phases in this compound. We outline an approximated method to compute the determinantal equation.

Subject Classification: $64.70 . \mathrm{Rh} ; 77.80 . \mathrm{Bh} ; \mathrm{S} 1.63$

\section{Introduction}

Many experimental and theoretical works with chargedensity-waves $(C D W)$ phase transitions in layered dichalcogenide transition metals, like $2 \mathrm{H}-\mathrm{TaSe} 2$, have emphasized the importance of the phenomenological McMillan-Landau theory [1] -[8], which was successful in introducing the notion of discommensuration defect or soliton-like behaviour of the incommensurate CDW near the incommensurate-commensurate 'lock-in' phase transition, despite the existence of alternative theoretical models[9]. The compound $2 \mathrm{H}-\mathrm{TaSe}_{2}$ has a layered structure; in each layer a hexagonal sheet of $T a$ ions is sandwiched between two hexagonal sheets of Se[10] ions. Moncton et al[11], from the initial neutron diffraction study found, as the temperature is lowered, an initial onset phase transition to an incommensurate $C D W$ phase at $122^{\circ} \mathrm{K}$ followed by another phase transition to a commensurate $C D W$ phase at $90^{\circ} \mathrm{K}$.

The order parameter fluctuation modes for the charge-density-waves in incommensurate systems have been studied by various authors and, in the case of $2 \mathrm{H}-\mathrm{TaSe}_{2}$, there has been a great deal of work due to the complexity of its phase transition picture. The understanding of the incommensurate superlattice of this substance, has been improved following the experimental work performed by Steinitz and Genossar[12] who inferred that in the range between $122^{\circ} \mathrm{K}$ and $90^{\circ} \mathrm{K}$, this superlattice presents two different phases. The first one with hexagonal symmetry exhibits at $112^{\circ} \mathrm{K}$ a firstorder phase transition to an orthorhombic stripe phase, which subsequently 'locks-in' at $90^{\circ} \mathrm{K}$. Chen at al[13] have stressed that 'on cooling', X-ray diffraction studies show that the $C D W$, although incommensurable, remains hexagonal and it is generally believed that a honeycomb array of narrow discommensurations[5] can also describe the incommensurate phase. Despite this possibility those authors have admitted that the hexagonal symmetry in the incommensurate superlattice of this compound is retained on a larger scale. Other writers [9], [14] have introduced an alternative model, where they have proposed three types $(I, I I, I I I)$ of orthorhombic stripe incommensurate $C D W$ phases, as well three types $(I, I I, I I I)$ of double honeycomb domain structure for the high-temperature incommensurate superlattice of a single layer having the $2 \mathrm{H}-\mathrm{TaSe}_{2}$ structure. Although we do not study this model here, we emphasize that these three distinct types of incommensurate phases correspond to three types $(I, I I, I I I)$ of commensurate phases of a single layer of this compound. There is experimental evidence that both the type- $I$ and type- $I I$ commensurate phases occur in this substance [11], [15]. From this approach only the typeI can be obtained from McMillan's phenomenological 
model. Those authors [14] have also showed how the layers could be stacked to give both hexagonal and orthorhombic structures.

In spite of these new theoretical approaches, our interests, in this work, have been in following McMillan's model, which is simpler and describes very well the distinct incommensurate and commensurate phases of this substance. In section II will perform calculations involving the commensurate $C D W$ phase, maintaining the early notation [7], [8], outlining eigenmode frequencies calculations in the hexagonal commensurate $C D W$ phase, comparing these results with those, of incommensurate $C D W$ phase, obtained previously [7] and providing new analytical results, a little different from ones of McMillan [8] because he omitted the contribution of the term, in his free energy expression, that computes the amplitude fluctuation modes (or amplitudons). That writer only discussed phase fluctuation modes (or phasons). In section III we present some analytical calculations of the eigenmode frequencies $\left(E_{2 g}, A_{1 g}\right)$ for the range of temperature decreasing from $122^{\circ} \mathrm{K}$, performing a limited numerical illustration and using a set of phenomenological parameters[7], in order to fit the two dominating modes, mentioned above, reported by Steigmeier et al[16], in his Raman scattering studies. They observed two dominating modes at
$49 \mathrm{~cm}^{-1}\left(E_{2 g}\right)$ and $82 \mathrm{~cm}^{-1}\left(A_{1 g}\right)$, at $6^{\circ} \mathrm{K}$, which were also confirmed by Sugai et al [17].

\section{Theory}

Let us consider the hexagonal commensurate phase of $2 \mathrm{H}-\mathrm{TaSe}_{2}$, which is characterized by the space group $D_{6 h}-6 / \mathrm{mmm}$. The McMillan-Landau free energy density [8] presents, in this case, a cubic"lock-in" energy term. This term is associated with the strong contribution to the lattice potential energy from the electrons due to the Peierls energy gap in band structure[18].

Now following the standard procedure we expand the order parameter as[7]

$$
\psi_{j}(\vec{r})=\phi_{j}(\vec{r}) \exp \left(i \vec{q}_{j} \cdot \vec{r}\right)
$$

$(j=1,2,3)$, where in the presence of excitations

$$
\phi_{j}(\vec{r})=\phi_{j}+\sum_{q} \phi_{j q} \exp (i \vec{q} \cdot \vec{r})
$$

$(j=1,2,3)$ and the $\vec{q}_{j}$ are the static charge-densitywave vectors. With $\phi_{1}=\phi_{2}=\phi_{3}=\phi_{0}$ and knowing that in this case $q_{j}=\frac{1}{3} \kappa_{j}$, being $\kappa_{j}$ the shortest reciprocal lattice vectors in the hexagonal symmetry, we write the new McMillan-Landau free energy for the hexagonal commensurate phase as

$$
\begin{aligned}
V= & \frac{1}{2} \int d^{2} r\left\{\sum _ { j } \left[a_{o}\left|\phi_{j}\right|^{2}+\frac{1}{4} 3 c_{o}\left|\phi_{j}\right|^{4}+2 e_{o}\left|\vec{Q}_{j} \cdot\left[\vec{\nabla}+i\left(\frac{1}{3} \vec{\kappa}_{j}-\vec{Q}_{j}\right)\right] \phi_{j}\right|^{2}\right.\right. \\
& \left.+2 f_{o}\left|\vec{Q}_{j} \times \overrightarrow{\nabla \phi_{j}}\right|^{2}\right]-\frac{1}{2} \operatorname{Re}\left(b_{1} \phi_{j}^{3}\right)-\frac{1}{2} 3 b_{o}\left(\phi_{1} \phi_{2} \phi_{3}+\phi_{1}^{*} \phi_{2}^{*} \phi_{3}^{*}\right) \\
& \left.+\left(3 c_{o}+2 d_{o}\right)\left(\left|\phi_{1} \phi_{2}\right|^{2}+\left|\phi_{2} \phi_{3}\right|^{2}+\left|\phi_{3} \phi_{1}\right|^{2}\right)\right\}
\end{aligned}
$$

where we see the contribution of the cubic 'lock-in' energy term, which is present in the mentioned phase. In order to maintain a comparison between the notation used by Holy et al[18] and McMillan[8] papers with our expression above, we note the following correspondence with their parameters and variables: $a_{o} \equiv A ; b_{1} \equiv 2 B=2 B_{l} \exp \left[i 3\left(\overrightarrow{q_{j}}\right.\right.$ $-\vec{G}) \cdot \vec{x}] ;\left(3 c_{o}+2 d_{o}\right) \equiv D ; b_{o} \equiv-\frac{1}{3} E=\frac{1}{3} \operatorname{Re}\left[\tilde{E} \exp \left(i 3 \theta_{o}\right)\right]$, and $f_{j} \equiv \phi_{l}^{j}=\phi_{l}^{j}$. The minimization of the static free energy with respect to $\phi_{0}$ gives

$$
2 a_{o}-3 b_{\circ} \phi_{o}-\frac{1}{2} 3 b_{1} \phi_{o}+15 c_{o} \phi_{o}^{2}+8 d_{o} \phi_{o}^{2}+4 e_{o}\left[Q_{1}^{2}\left(\frac{1}{3} \kappa_{1}-Q_{1}\right)^{2}\right]=0
$$

with $Q_{1}=\left|\vec{Q}_{i}\right|$ and $\kappa_{1}=\left|\vec{\kappa}_{i}\right|$. 
Now we compute the phonon frequencies and we use the expansion (2), such that this procedure corresponds to the addition of small phase and amplitude distortions to static distortion. Using Eq. (4) we expand (3) in powers of $\phi_{j q}$ from (2) and keeping only second order terms, and following McMillan's procedure we compute the eigenmodes, writing in terms of the coupled modes and find

$$
V=V\left(\phi_{0}\right)+V^{+}+V^{-}+V^{ \pm}
$$

where

$$
\begin{aligned}
V\left(\phi_{o}\right)= & \frac{1}{2} 3\left\{a_{o}-\left(b_{o}+\frac{1}{2} b_{1}\right) \phi_{o}+\left(\frac{1}{4} 15 c_{o}+2 d_{o}\right) \phi_{o}^{2}+2 e_{o}\left[Q_{1}^{2}\left(\frac{1}{3} \kappa_{1}-Q_{1}\right)^{2}\right]\right\} \phi_{o}^{2} \\
V^{+}= & \frac{1}{2}\left\{\sum_{j q}\left[\frac{1}{4} 3 b_{o} \phi_{o}-\frac{1}{8} 3 b_{1} \phi_{o}+\frac{1}{2} 3 c_{o} \phi_{o}^{2}+e_{o}\left(\overrightarrow{Q_{j}} \cdot \vec{q}\right)^{2}+f_{o}\left(\overrightarrow{Q_{j}} \times \vec{q}\right)^{2}\right] A_{j q}^{*} A_{j q}\right. \\
& \left.-\left[\frac{1}{4} 3 b_{o} \phi_{o}-\left(3 c_{o}+2 d_{o}\right) \phi_{o}^{2}\right] \sum_{q}\left(A_{1 q}^{*} A_{2 q}+A_{2 q}^{*} A_{1 q}+\text { permutations }\right)\right\} \\
V^{-} & \frac{1}{2}\left\{\sum_{j q}\left[\frac{1}{4} 3 b_{o} \phi_{o}+\frac{1}{8} 9 b_{1} \phi_{o}+e_{o}\left(\overrightarrow{Q_{j}} \cdot \vec{q}\right)^{2}+f_{o}\left(\overrightarrow{Q_{j}} \times \vec{q}\right)^{2}\right] P_{j q}^{*} P_{j q}\right. \\
& \left.+\frac{1}{4} 3 b_{o} \phi_{o} \sum_{q}\left(P_{1 q}^{*} P_{2 q}+P_{2 q}^{*} P_{1 q}+\text { permutations }\right)\right\} \\
V^{ \pm} & =\frac{1}{2} e_{o} Q_{1}\left(\frac{1}{3} \vec{\kappa}_{1}-\vec{Q}_{1}\right) \sum_{j q} 2\left(\overrightarrow{Q_{j}} \cdot \vec{q}\right)\left(A_{j q}^{*} P_{j q}+P_{j q}^{*} A_{j q}\right)
\end{aligned}
$$

In order to avoid some difficulties in solving the complete $6 \times 6$ characteristic determinantal equation to compute the fluctuation eigenmodes, we have only considered the particular situation where $\vec{q}$ is parallel to $\vec{Q}_{1}$. Such situation results from the contribution of the cross q-linear term given by Eq. (9) above, which does not occur in the hexagonal incommensurate case[7]. Performing some algebraic manipulations, we are able to rewrite the original matrix as the product of two other matrices with dimensions $4 \times 4$ and $2 \times 2$ respectively. The advantage of this calculation is that the $2 \times 2$ determinantal equation gives directly expressions for the $E_{2 g}$ and $E_{1 u}$ eigenmode frequencies; and the $4 \mathrm{x}$ 4 matrix provides the eigenmodes $E_{2 g}, A_{1 g}, E_{1 u}, B_{1 u}$, avoiding the degeneracy problem of the doublet amplitudons or phasons respectively. With this procedure, we find the following results,

$$
E_{2 g}: E_{2 g}^{o}+\frac{1}{4}\left(e_{o}+3 f_{o}+4 e_{o}^{2} \nu E_{2 g}^{o-1}\right) Q_{1}^{2} q^{2}
$$

$$
E_{1 u}: \frac{1}{8} 9 b_{1} \phi_{o}+\frac{1}{4}\left(e_{o}+3 f_{o}-4 e_{o}^{2} \nu E_{2 g}^{o-1}\right) Q_{1}^{2} q^{2}
$$

where we have defined

$$
\begin{gathered}
E_{2 g}^{o}=\frac{1}{2} 3\left(b_{o}-\frac{1}{4} b_{1}\right) \phi_{o}-\left(\frac{1}{2} 3 c_{o}+2 d_{o}\right) \phi_{o}^{2} \\
\nu=\left(\frac{1}{3} \kappa_{1}-Q_{1}\right)^{2} Q_{1}^{2}
\end{gathered}
$$

Equation (12) gives the frequencies of the modes with symmetry $E_{2 g}$ at $q=0$, and we also see that the quadratic contribution in $e_{0}$ results from the cross q-linear term. As we have written before[7] the expressions (10) and (11) represents $\frac{M^{*} \omega^{2}}{4}$, being $\omega$ the mode frequency and $M^{*}=206 a u$. $E_{2 g}$ and $E_{1 u}$ are the symmetry characters of the irreducible representations of the group $D_{6 h}-6 / \mathrm{mmm}$.

Our 4 x 4 determinantal equation can be written as 


\section{Numerical Results}

$$
\left|\begin{array}{llll}
a-\lambda & 2 c & -2 d & 0 \\
c & b+c-\lambda & 0 & d \\
-2 d & 0 & a^{\prime}-\lambda & 2 c^{\prime} \\
0 & d & c^{\prime} & b^{\prime}+c^{\prime}-\lambda
\end{array}\right|=0
$$

where we have defined: $a=\frac{1}{4} 3\left(b_{o}-\frac{1}{2} b_{1}\right) \phi_{o}+\frac{1}{2} 3 c_{o} \phi_{o}^{2}+$ $e_{o} Q_{1}^{2} q^{2} ; a^{\prime}=\frac{1}{4} 3\left(b_{\circ}+\frac{1}{2} 3 b_{1}\right) \phi_{\circ}+e_{o} Q_{1}^{2} q^{2} ; b=\frac{1}{4} 3\left(b_{\circ}-\right.$ $\left.\frac{1}{2} b_{1}\right) \phi_{o}+\frac{1}{2} 3 c_{o} \phi_{o}^{2}+\frac{1}{4}\left(e_{o}+3 f_{o}\right) Q_{1}^{2} q^{2} ; b^{\prime}=\frac{1}{4} 3\left(b_{o}+\right.$ $\left.\frac{1}{2} 3 b_{1}\right) \phi_{o}+\frac{1}{4}\left(e_{o}+3 f_{0}\right) Q_{1}^{2} q^{2} ; c=-\frac{1}{4} 3 b_{o} \phi_{o}+\left(3 c_{o}+2 d_{o}\right) \phi_{o}^{2}$; $c^{\prime}=\frac{1}{4} 3 b_{o} \phi_{o} ; d=-e_{o}\left(\frac{1}{3} \kappa_{1}-Q_{1}\right) Q_{1}^{2} q$.

In these calculations we see from $V^{+}, V^{-}$and $V^{ \pm}$ expressions that $a, b, c$, are directly connected with amplitudons; $a^{\prime}, b^{\prime}, c^{\prime}$, with phasons, and $d$ with the cross term.

In reality the exact solution of the determinantal equation (14) is not straightforward and we have used an approximated method in order to calculate the last four non-degenerate fluctuation eigenmodes. We can outline this procedure rewriting that equation as

$\left(\lambda-\lambda_{1}\right)\left(\lambda-\lambda_{2}\right)\left(\lambda-\lambda_{3}\right)\left(\lambda-\lambda_{4}\right)-\left(A \lambda^{2}+B \lambda+C\right) q^{2} \sim 0$

being $\lambda_{i}$ the eigenvalues and $A, B$ and $C$ are expressed in terms of $a, a^{\prime}, b, b^{\prime}, \ldots, d$, as we will discuss in the appendix. We will derive the following expression for the four eigenvalues,

$$
\lambda_{j}^{\prime}=\lambda_{j}+\frac{A \lambda_{j}^{2}+B \lambda_{j}+C}{\left(\lambda_{j+1}-\lambda_{j}\right)\left(\lambda_{j+2}-\lambda_{j}\right)\left(\lambda_{j+3}-\lambda_{j}\right)} q^{2}
$$

where $j=1,2,3,4$ and $\lambda_{5}=\lambda_{1}, \lambda_{6}=\lambda_{2}$ and $\lambda_{7}=\lambda_{3}$. Despite this expression is not evaluated here, meanwhile it indicates how the analytical expression can be obtained for the eigenvalues with symmetries $A_{1 g}, E_{2 g}, B_{1 u}, E_{1 u}$, respectively, to order $q^{2}$. We do not consider the case of $\vec{q}$ perpendicular to $\vec{Q}_{1}$, because this has more severe algebraic difficulties, and we cannot obtain similar simplification. If we consider the particular case of $q=0$ the determinantal equation (14) can be solved to obtain the following expression: $A_{1 g}:-\frac{1}{4} 3\left(b_{o}+\frac{1}{2} b_{1}\right) \phi_{o}+\frac{1}{2}\left(15 c_{o}+8 d_{o}\right) \phi_{o}^{2} ; E_{2 g}$ : $\frac{1}{2} 3\left(b_{o}-\frac{1}{4} b_{1}\right) \phi_{o}-\frac{1}{2}\left(3 c_{o}+4 d_{o}\right) \phi_{o}^{2} ; B_{1 u}: \frac{1}{4} 9\left(b_{o}+\frac{1}{2} b_{1}\right) \phi_{o}$; $E_{1 u}: \frac{1}{8} 9 b_{1} \phi_{o}$. These last expressions are similar in outline but differ in detail from those reported by Holy at al[18]. We must stress that it is not possible to make a complete comparison because those authors used a different free energy for their commensurate phase calculations in $2 \mathrm{H}-\mathrm{TaSe}_{2}$.
In this section we present the results of a numerical illustration of eigenmode frequencies $\left(E_{2 g}, A_{1 g}\right)$ of $2 \mathrm{H}-\mathrm{TaSe}_{2}$. We have used the normalized coordinates $\tilde{\omega}=\frac{1}{2} M^{* \frac{1}{2}} \omega$ and $\tilde{q}=e_{o}^{\frac{1}{2}} Q_{1} q$ defined previously [7], and we have assumed the phenomenological parameters $b_{o}=\frac{4}{30}, b_{1}=1.2, c_{0}=\frac{8}{3}, d_{o}=-3.9$ and $f_{o}=0$. We have used a quadratic expression, $a_{0}=a^{\prime}\left(T^{2}-T_{o}^{2}\right)$, as the definition of Landau parameter $a_{0}$ in terms of temperature, with $a^{\prime}=2.35 \times 10^{-4}$. If the standard Landau expression, $a_{0}=a^{\prime}\left(T-T_{0}\right)$, is used at low temperatures regime, we find that the order parameter and mode frequencies have a linear component in their dependence temperature as $T \rightarrow 0$, in violation of the third law of Thermodynamics[7]. Our quadratic expression has the usual linear form for $T$ close to $T_{0}$ but it also produces the thermodynamically correct behaviour as $T \rightarrow 0$. For the case of the hexagonal commensurate phase, there is a visible variation of the frequencies of these optical phonons with temperature but it is not strongly.

We must emphasize that our calculations are in fact an approximation to the exact ones, because the analytical calculations involving the normal mode frequencies in this commensurate phase are not straightforward, due to the cross $q$-linear term $V^{ \pm}$. We have also performed numerical computations in the region of the incommensurate-commensurate "lock-in " phase transition, obtaining the order parameter's behavior as the temperature changes over the complete range between $122^{\circ} \mathrm{K}$ and $0^{\circ} \mathrm{K}$. We consider the experimental 'on cooling' situation, where the orthorhombic stripe incommensurate phase for this compound does not occur, that is, in the range of temperature between $122^{\circ} \mathrm{K}$ and $90^{\circ} \mathrm{K}$, the unique phase is the hexagonal incommensurate phase.

Let as consider the equations of minimization of the static free energy for the incommensurate case [7] with respect to $\phi_{0}$

$$
2 a_{o}-3 b_{o} \phi_{o}+\left(15 c_{o}+8 d_{o}\right) \phi_{o}^{2}=0
$$

and the similar for the commensurate case (4) that give the analytical expressions for the order parameter for the hexagonal incommensurate $\left(\phi_{\text {inc }}\right)$ and commensurate $\left(\phi_{c}\right)$ phases respectively. Then, using the new definition of the Landau parameter $a_{o}$, we rewrite $V\left(\phi_{0}\right)$ for the case of both phases as, 


$$
V_{i n c}^{o}=\frac{1}{2} 3\left[a_{o}-b_{o} \phi_{i n c}+\left(\frac{1}{4} 15 c_{o}+2 d_{o}\right) \phi_{i n c}^{2}\right] \phi_{i n c}^{2}
$$

and

$$
V_{c}^{o}=\frac{1}{2} 3\left[a_{o}-\left(b_{o}+\frac{1}{2} b_{1}\right) \phi_{c}+\left(\frac{1}{4} 15 c_{o}+2 d_{o}\right) \phi_{c}^{2}+\frac{1}{2} e^{\prime}\right] \phi_{c}^{2}
$$

where $e^{\prime}$ represents $4 e_{o}\left[Q_{1}^{2}\left(\frac{1}{3} \kappa_{1}-Q_{1}\right)^{2}\right]$.

The numerical problem is to find a set of phenomenological parameters that produce the incommensurate-commensurate "lock-in" transition temperature at $90^{\circ} \mathrm{K}$, that is, the point where $V_{i n c}^{o}=$ $V_{c}^{o}$. To illustrate this procedure, we have used the same values of $b_{o}, b_{1}, c_{o}$ and $d_{o}$ introduced by Ribeiro Filho[7] and take $e^{\prime}=0.779$. The next step is to solve numerically the equation $V=V_{c}^{o}(T)-V_{\text {inc }}^{o}(T)=0$, $T$ being the "lock-in" temperature. These calculations can be performed when we substitute Eqs. (17) and (4) in $V_{i n c}^{o}$ and $V_{c}^{o}$, respectively. The expression for $V$ is an equation $V\left(a^{\prime}\right)=0$ that gives the values of $a^{\prime}$ for a particular set of phenomenological parameters. If we use the parameter mentioned above we obtain $a^{\prime}=2.35 \times 10^{-4}$. Figs. (1) and (2) show the free energies $V^{o}$ relative to the incommensurate and commensurate phases and the order parameter $\left(\phi_{0}\right)$ versus temperature for $2 \mathrm{H}-\mathrm{TaSe}_{2}$. Further numerical computations have been made in order to fit the theoretical results for eigenmode frequencies to light scattering data. We have concentrated on the experimental results of Raman scattering reported by Steigmeier et al [16], that are an important contribution to the understanding of the commensurate charge-density-wave phase of $2 \mathrm{H}-\mathrm{TaSe}_{2}$. They observed two dominating modes at $49 \mathrm{~cm}^{-1}\left(E_{2 g}\right)$ and $82 \mathrm{~cm}^{-1}\left(A_{1 g}\right)$, at $6^{\circ} \mathrm{K}$, which were confirmed by Sugai et al [17]. We have performed a limited numerical calculation of these two eigenmode frequencies $\left(E_{2 g}, A_{1 g}\right)$, normalizing the Steigmeier et al results to $T=0^{\circ} \mathrm{K}$. We retain the same values for parameters $b_{o}, b_{1}, c_{o}, d_{o}$ and calculate $a^{\prime}$ and $e^{\prime}$ algebraically, with $a_{o}$ defined before. Then, after some straightforward manipulations with the order parameter and frequency expression of $A_{1 g}$ and $E_{2 g}$, we write for the range of temperature between $0^{\circ} \mathrm{K}$ and $90^{\circ} \mathrm{K}$,

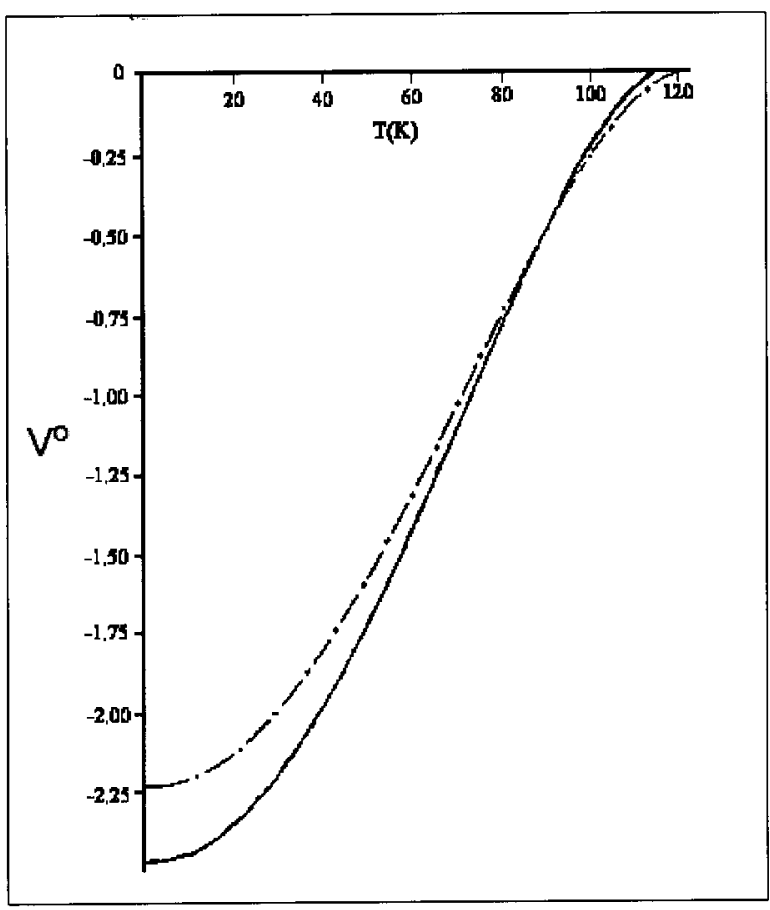

Figure 1. Free energies, relative to the incommensurate $(--\bullet)$ and commensurate $(-)$ phase for a single layer of the $2 \mathrm{H}-\mathrm{TaSe}_{2}$ as a function of temperature with all parameters given in the text.

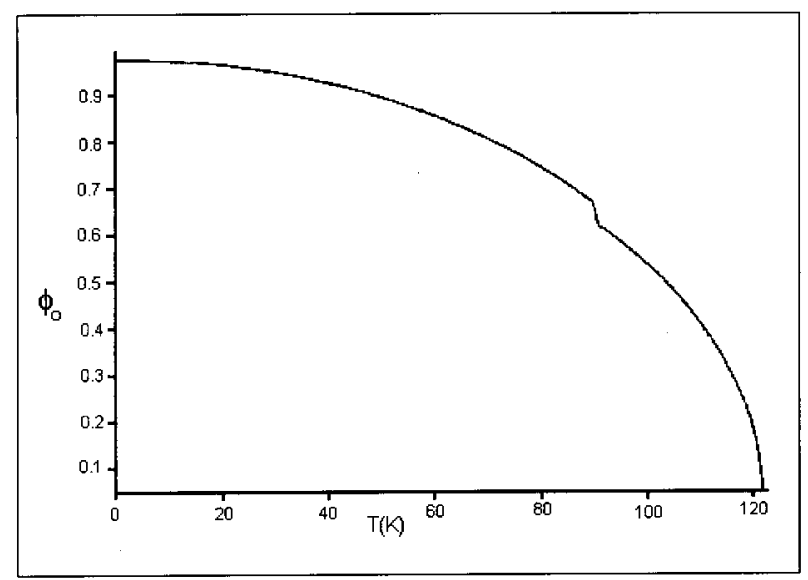

Figure 2. Order parameter $\left(\phi_{0}\right)$ versus temperature for $2 \mathrm{H}-$ $\mathrm{TaSe}_{2}$, with all parameters given in the text. 


$$
\omega_{A_{1 g}}(T)=\omega_{A_{1 g}}(0)\left[\left\{\left[\omega_{A}^{\prime}+\omega_{A} \phi_{c}(T)\right] \phi_{c}(T)\right\}\left\{\left[\omega_{A}^{\prime}+\omega_{A} " \phi_{c}(0)\right] \phi_{c}(0)\right\}^{-1}\right]^{\frac{1}{2}}
$$

where $\omega_{A}^{\prime}=-\frac{1}{4} 3\left(b_{\circ}+\frac{1}{2} b_{1}\right)$ and $\omega_{A} "=\frac{1}{2}\left(15 c_{o}+8 d_{o}\right)$; and

$$
\omega_{E_{2 g}}(T)=\omega_{E_{2 g}}(0)\left[\left\{\left[\omega_{E}^{\prime}+\omega_{E}^{\prime \prime} \phi_{c}(T)\right] \phi_{c}(T)\right\}\left\{\left[\omega_{E}^{\prime}+\omega_{E} " \phi_{c}(0)\right] \phi_{c}(0)\right\}^{-1}\right]^{\frac{1}{2}}
$$

where $\omega_{E}^{\prime}=\frac{1}{2} 3\left(b_{o}-\frac{1}{4} b_{1}\right)$ and $\omega_{E} "=-\frac{1}{2}\left(3 c_{o}+4 d_{o}\right)$; with $\omega_{A_{1 g}}(0)$ and $\omega_{E_{2 g}}(0)$ being the experimental values of Steigmeier et al, and $\phi_{e}(T)$ has been obtained from the minimization of the static commensurate free energy.

In order to get an approximate picture of these modes for the incommensurate phase [7], we have written for the range between $90^{\circ} \mathrm{K}<T<122^{\circ} \mathrm{K}$, the eigenmode frequencies as

$$
\omega_{A_{1 g}}(T)=\omega_{A_{1 g}}(90)\left[\left\{\left[-\frac{1}{4} 3 b_{o}+\omega_{A}^{\prime \prime} \phi_{\text {inc }}(T)\right] \phi_{\text {inc }}(T)\right\}\left\{\left[-\frac{1}{4} 3 b_{o}+\omega_{A}{ }^{\prime} \phi_{\text {inc }}(90)\right] \phi_{\text {inc }}(90)\right\}^{-1}\right]^{\frac{1}{2}}
$$

and

$$
\omega_{E_{2 g}}(T)=\omega_{E_{2 g}}(90)\left[\left\{\left[\frac{1}{2} 3 b_{o}+\omega_{E}^{\prime} \phi_{\text {ine }}(T)\right] \phi_{\text {inc }}(T)\right\}\left\{\left[\frac{1}{2} 3 b_{o}+\omega_{E} " \phi_{\text {ine }}(90)\right] \phi_{\text {ine }}(90)\right\}^{-1}\right]^{\frac{1}{2}}
$$

Equations (20-23) are plotted in Fig. (3) and give a reasonable picture of the Steigmeier et al experimental work $(1976)$.

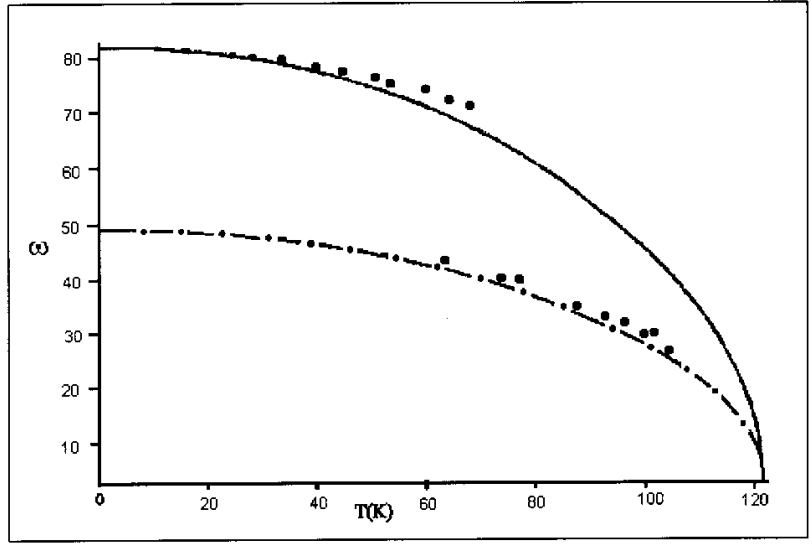

Figure 3. Frequencies of the CDW models of $2 \mathrm{H}-\mathrm{Ta} \mathrm{Se}_{2}$ versus temperature. Fitting from Steigmeier et all for $\mathrm{A}_{1 \mathrm{~g}}$ $(--)$ and $\mathrm{E}_{2 g}(--\bullet)$ modes, with all parameters given in the text.

\section{Conclusions}

In this work we have reported calculations of eigenmode frequencies for an assumed hexagonal commensurate charge-density-wave phase of $2 \mathrm{H}-\mathrm{TaSe}_{2}$, despite the controversy whether such phase is orthorhombic [6] rather than hexagonal [11]. Fung et al (1981) found that the commensurate phase is not hexagonal but rather a network of orthorhombic domains and that the hexagonal symmetry observed in scattering experiments was due to domain averaging. Another interesting contribution to the orthorhombic-hexagonal controversy has been given by Walker and Jacobs[14] that introduced an alternative free energy functional for the $C D W$ states in $2 \mathrm{H}-\mathrm{TaSe}_{2}$. Those writers have classified three distinct $(I, I I, I I I)$ commensurate $C D W$ states with hexagonal (type- $I$ layers) and orthorhombic (type- II, III layers) symmetries. In fact the experimental study of the incommensurate and commensurate superlattices of this compound has been intense during recent years, and there is the possibility that new symmetry's interpretations can be improved in the next future.

We have also performed some numerical computations in order to give an approximated picture of some experimental results for this compound. The analytical results for commensurate $C D W$ phase can be correlated with those ones of the incommensurate phase[7]. 
We have assumed 'on-cooling' experimental situation where only the hexagonal incommensurate phase is present in the range of temperature between $122^{\circ} \mathrm{K}$ and $90^{\circ} \mathrm{K}$. In the case of the commensurate superlattice we have neglected any orthorhombic symmetry, retaining the original picture of the hexagonal symmetry existing throughout the commensurate phase. In spite of the limitations of this numerical illustration, it shows the advantage of the McMillan-Landau theory in providing a unified description of the charge-density-wave phases of this compound. We have written some normalized expressions for eigenmode frequencies in order to fit the Raman scattering $\left(E_{2 g}, A_{1 g}\right)$ data obtained by Steigmeier et al[16]. It is important to stress that these two previously identified hexagonal $E_{2 g}$ modes can be then be thought of as superpositions of $A_{g}$ and $B_{l g}$ orthorhombic modes, while the two previously identified $A_{l g}$ modes become $A_{g}$ orthorhombic modes[19]. These correlations between the two symmetry possibilities are very interesting because they create a link between the experimental results of those authors that found hexagonal or orthorhombic symmetries. Finally, we must emphasize that we have obtained, from Fig. 3, a reasonable fitting from the Raman scattering data obtained by Steigmeier et al.

Acknowledgment One of us (A. R. F.) would like to thank the support of "Conselho Nacional de Desenvolvimento Cientifíco e Tecnológico" ( $\mathrm{CNPq})$ and the Brazilian agency CAPES through the "CDI-Programa Suplementar de Apoio à Qualificação Docente".

\section{References}

[1] J. F. M. Rocha, D. S. de Vasconcelos and A. Ribeiro Filho, Phys. Stat. Sol. (b) 205, 473 (1998).
[2] S. A. Jackson, P. A. Lee and T. M. Rice, Phys. Rev. B17, 9, 3611 (1978).

[3] T. M. Rice, Phys. Rev. B23, 5, 2413 (1981).

[4] K. Nakanishi, H. Takatera, Y. Yamada and H. Shiba, J. Phys. Soc. Jap. 43, 5, 1509 (1977).

[5] K. Nakanishi and H. Shiba, J. Phys. Soc. Jap. 43, 6, 1839 (1977); 44, 5, 1965 (1978).

[6] K. K. Fung, S. McKernan, J. W. Steeds and J. A. Wilson, Phys. Rev. B14, 5417 (1981).

[7] A. Ribeiro Filho, Rev. Bras. de Física (now Braz. J. Phys.) 16, 233 (1986); 16, 240 (1986); PhD Thesis, University of Essex, U. K. (1984).

[8] W. L. McMillan, Phys. Rev. B12, 1187, 1197 (1975); B14, 1496 (1976); B16, 543 (1977); B16, 4655 (1977).

[9] A. E. Jacobs and M. B. Walker, Phys. Rev. B26, 206 (1982).

[10] J. A. Wilson and A. D. Yoffe, Adv. Phys. 18, 193 (1969).

[11] D. E. Moncton, J. D. Axe and F. J. Di Salvo, Phys. Rev. B16, 801 (1977).

[12] M. O. Steinitz and J. G-Genossar, Sol. Stat. Comm., 29, 519 (1979).

[13] C. H. Chen, J. M. Gibson and R. M. Fleming, Phys. Rev. B26, 1, 184 (1982).

[14] M. B. Walker and A. E. Jacobs, Phys. Rev. B24, 6770 (1981).

[15] B. H. Suits, S. Conturie and C. P. Slichter, Phys. Rev. B23, 10, 5142 (1981).

[16] E. F. Steigmeier, J. Harbeke, H. Auderset and F. J. Di Salvo, Sol. Stat. Comm. 20, 667 (1976); In Electron Phonon Interactions and Phase Transitions, ed. by T. Riste (1977).

[17] S. Sugai, K. Murase, S. Ushida and S. Tanaka, Physica 105B, 405 (1981).

[18] J. A. Holy, M. V. Klein, W. L. McMillan, and S. F. Meyer, Phys. Rev. Lett. 37, 17, 1145 (1976).

[19] R. L. Withers and M. B. Walker, Phys. Rev. B26, 2, 940 (1982).

\section{Appendix}

In this section we discuss the calculation of eigenmodes from the determinantal equation (14) using an approximated method outlined below. We rewrite it as

$$
\left|\begin{array}{cccc}
a-\lambda & 2 c & -2 d & 0 \\
c & b+c-\lambda & 0 & d \\
-2 d & 0 & a^{\prime}-\lambda & 2 c^{\prime} \\
0 & d & c^{\prime} & b^{\prime}+c^{\prime}-\lambda
\end{array}\right|=0
$$

with $a, a^{\prime}, b, b^{\prime}, c, c^{\prime}, d$, as defined before. After performing straightforward algebraic manipulations, we write Eq. (24) as 


$$
A \cdot P+\left[8 c c^{\prime}-(a-\lambda)\left(a^{\prime}-\lambda\right)+4(b+c-\lambda)\left(b^{\prime}+c^{\prime}-\lambda\right)\right] d^{2} \sim 0
$$

where

$$
A=\left|\begin{array}{cc}
a-\lambda & 2 c \\
c & b+c-\lambda
\end{array}\right|
$$

and

$$
P=\left|\begin{array}{cc}
a^{\prime}-\lambda & 2 c^{\prime} \\
c^{\prime} & b^{\prime}+c^{\prime}-\lambda
\end{array}\right|
$$

where we have neglected the quartic d-contribution. In order to get approximate solution of (25), we consider first the case when $d$ vanishes in (24). So, we have two uncoupled $2 \times 2$ determinantal equations $A=0$ and $P=0$, which after diagonalization could be written as

$$
\left(\lambda_{1}-\lambda\right)\left(\lambda_{2}-\lambda\right)\left(\lambda_{3}-\lambda\right)\left(\lambda_{4}-\lambda\right)=0
$$

In this case, using the same procedure outlined before and substituting $a, a^{\prime}, b, b^{\prime}, c, c^{\prime}, d$, in (26) and (27) we find

$$
\begin{gathered}
\lambda_{1}=-\frac{1}{4} 3\left(b_{o}+\frac{1}{2} b_{1}\right) \phi_{o}+\left(\frac{1}{2} 15 c_{o}+4 d_{o}\right) \phi_{o}^{2}+\frac{1}{2}\left(e_{o}+f_{o}\right) Q_{1}^{2} q^{2} \\
\lambda_{2}=\frac{1}{2} 3\left(b_{o}-\frac{1}{4} b_{1}\right) \phi_{o}-\left(\frac{1}{2} 3 c_{o}+2 d_{o}\right) \phi_{o}^{2}+\frac{1}{4}\left(3 e_{o}+f_{o}\right) Q_{1}^{2} q^{2} \\
\lambda_{3}=\frac{1}{4} 9\left(b_{o}+\frac{1}{2} b_{1}\right) \phi_{o}+\frac{1}{2}\left(e_{o}+f_{o}\right) Q_{1}^{2} q^{2} \\
\lambda_{4}=\frac{1}{8} 9 b_{1} \phi_{o}+\frac{1}{4}\left(3 e_{o}+f_{o}\right) Q_{1}^{2} q^{2}
\end{gathered}
$$

If we compare (29-32) with the expressions of $A_{1 g}, E_{2 g}, B_{1 u}$ and $E_{1 u}$, given in section II, we identify these modes as follows: $\lambda_{1} \rightarrow A_{1 g} ; \lambda_{2} \rightarrow E_{2 g} ; \lambda_{3} \rightarrow B_{1 u}$; and $\lambda_{4} \rightarrow E_{1 u}$. When we consider $d \neq 0$ in (25) we retain only terms to order $q^{2}$. After some algebraic manipulation we can rewrite (25) as

$$
\left(\lambda-\lambda_{1}\right)\left(\lambda-\lambda_{2}\right)\left(\lambda-\lambda_{3}\right)\left(\lambda-\lambda_{4}\right)-\left(A \lambda^{2}+B \lambda+C\right) q^{2}=0
$$

where

$$
\begin{gathered}
A=5 e_{o}^{2}\left(\frac{1}{3} \kappa_{1}-Q_{1}\right)^{2} Q_{1}^{4} \\
B=-\frac{1}{2} e_{o}^{2}\left[15\left(b_{o}+\frac{1}{2} b_{1}\right)+\left(39 c_{o}+16 d_{o}\right)\right] \phi_{o}\left(\frac{1}{3} \kappa_{1}-Q_{1}\right)^{2} Q_{1}^{4} \\
C=9 e_{o}^{2}\left\{\frac{1}{2}\left(\frac{1}{2} b_{o} b_{1}+b_{o}^{2}-\frac{1}{8} 3 b_{1}^{2}\right)+\left[b_{o}\left(c_{o}+d_{o}\right)+\frac{1}{4} 9 b_{1} c_{o}\right] \phi_{o}\right\} Q_{1}^{2}\left(\frac{1}{3} \kappa_{1}-Q_{1}\right)^{2} Q_{1}^{4}
\end{gathered}
$$

Equation (33) produces for instance the approximate value $\lambda_{1}^{\prime}$ of the eigenmode $\lambda_{1}$, where $\lambda_{1}^{\prime}=\lambda_{1}+\varepsilon$ with $\varepsilon \sim O\left(q^{2}\right)$. In this case when we substitute $\lambda=\lambda_{1}^{\prime}$, we get

$$
\left(\lambda_{1}^{\prime}-\lambda_{1}\right)\left(\lambda_{1}^{\prime}-\lambda_{2}\right)\left(\lambda_{1}^{\prime}-\lambda_{3}\right)\left(\lambda_{1}^{\prime}-\lambda_{4}\right)-\left(A \lambda_{1}^{\prime 2}+B \lambda_{1}^{\prime}+C\right) q^{2}=0
$$

and to lowest order in $q^{2}$, 


$$
\lambda_{1}^{\prime}=\lambda_{1}+\frac{A \lambda_{1}^{2}+B \lambda_{1}+C}{\left(\lambda_{1}-\lambda_{2}\right)\left(\lambda_{1}-\lambda_{3}\right)\left(\lambda_{1}-\lambda_{4}\right)} q^{2}
$$

Following the same procedure, we can write a general expression for these eigenvalues:

$$
\lambda_{j}^{\prime}=\lambda_{j}+\frac{A \lambda_{j}^{2}+B \lambda_{j}+C}{\left(\lambda_{j+1}-\lambda_{j}\right)\left(\lambda_{j+2}-\lambda_{j}\right)\left(\lambda_{j+3}-\lambda_{j}\right)} q^{2}
$$

with $j=1,2,3,4$, and $\lambda_{5}=\lambda_{1}, \lambda_{6}=\lambda_{2}, \lambda_{7}=\lambda_{3}$. The values of $\lambda_{j}$ are given by equations (29-32). 